\title{
VALORES RELIGIOSOS EN PROFESIONALES DE ENFERMERÍA EGRESADOS DE UNIVERSIDADES DOMINICANAS
}

\section{RELIGIOUS VALUES IN NURSING PROFESSIONALS GRADUATED FROM DOMINICAN UNIVERSITIES}

\author{
Alfa R. Suero de Lora \\ Universidad Adventista Dominicana, República Dominicana \\ arsuerom@unad.edu.do \\ https://orcid.org/0000-0002-1416-7499 \\ Fermín Ferriol Sánchez
Universidad Internacional Iberoamericana, Puerto Rico
fermin.ferriol@unini.edu.mx
https://orcid.org/0000-0003-4138-8999

\begin{abstract}
RESUMEN
Se realizó un estudio mixto con el objetivo de analizar las reacciones de profesionales de enfermería hacia los valores religiosos, que puedan estar incidiendo en su actuación laboral. Se aplicó el Test de Reacción Valorativa a 203 enfermeros activos en centros de salud pública y privada de siete ciudades dominicanas. Además, se tomó nota de las expresiones observadas en los sujetos al completar la prueba. Se encontró que los valores religiosos se ubicaron en la última posición de la escala, con solo un $13 \%$ de profesionales en el máximo rango de puntuación (40-50 puntos), hallazgo que se mostró relacionado con un apego a las creencias religiosas aceptadas e indiferencia o rechazo de las creencias del otro. También se observó un conocimiento bajo de otras culturas. Se concluye que, siendo la República Dominicana un país eminentemente cristiano, donde se incrementa la diversidad cultural por fenómenos migratorios, existe la necesidad de integrar valores religiosos en la formación de estudiantes y profesionales de enfermería, en un marco de tolerancia y respeto, dada la diversidad que refleja en la demanda del servicio de salud. Finalmente, se concluye que deben realizarse estudios sobre la calidad del cuidado espiritual que se brinda en condiciones transculturales, sobre todo en la zona fronteriza.
\end{abstract}

Palabras clave: valores religiosos, cuidado espiritual, ética de enfermería.

\section{ABSTRACT}

A mixed study was carried out in order to analyze the reactions of nursing professionals towards religious values, which may be influencing their work performance. The Valuation Reaction Test was applied to 203 active nurses in public and private health centers in seven Dominican cities. In addition, the expressions observed in the subjects when completing the test 


\section{SUERO DE LORA Y FERRIOL SÁNCHEZ}

were noted. Religious values were found to be in the last position on the scale, with only 13\% of professionals in the maximum scoring range (40-50 points), a finding that was shown to be related to an attachment to accepted religious beliefs and indifference or rejection of the beliefs of the other. Low knowledge of other cultures was also observed. It is concluded that, since the Dominican Republic is an eminently Christian country, where cultural diversity increases due to migratory phenomena, there is a need to integrate religious values in the training of nursing students and professionals, within a framework of tolerance and respect, given the diversity reflected in the demand of the health service. Finally, it is concluded that studies should be carried out on the quality of spiritual care provided in cross-cultural conditions, especially in the border zone.

Keywords: religious values, spiritual care, nursing ethics, training of nurses

\section{Introducción}

La espiritualidad del paciente es un elemento que en las últimas décadas está siendo objeto de estudio, por haberse encontrado evidencias de su vinculación con la prevención y el afrontamiento de la enfermedad, así como con la recuperación de la salud (Okasha y Okasha, 2012; Puentes Silva et al., 2015). Las investigaciones han reportado que un paciente con sus necesidades espirituales atendidas tiende a recuperarse en menor tiempo y mantener una perspectiva positiva (Salgado, 2014; Yoffe, 2007). De ahí que la dimensión espiritual es tomada en cuenta dentro del paradigma científico de las profesiones que atienden la salud.

En el caso de la enfermería, el segundo principio del primer elemento del Código Deontológico Internacional, que establece la relación de la enfermera con la persona, aboga por el respeto a las creencias del paciente al declarar: "Al dispensar los cuidados, la enfermera promoverá un entorno en el que se respeten los derechos humanos, valores, costumbres y creencias espirituales de la persona, la familia y la comunidad" (Consejo Internacional de Enfermería, 2012, p. 3). Es un consenso al cual se ha llegado con años de estudio y a partir de que la comprensión de la realidad de este deber llevó al desarrollo de modelos de atención de enfermería con inclusión del cuidado espiritual de la persona, como los de Henderson (1961), Orem (1959) y Watson (2015).

Según Sessanna et al. (2007, citados en Sierra Leguía y Montalvo Prieto, 2012), dentro de la espiritualidad se deben atender cuatro dimensiones: (a) sistema religioso de creencias y valores, (b) vida, significado y conexión con otros, (c) sistema de creencias y valores no religiosos, y (d) fenómeno metafísico o trascendente. En ese conjunto, los valores religiosos muestran relevancia porque los otros tres factores están interrelacionados con ellos. Álvarez Rodríguez (2007) define los valores religiosos como aquellos que hacen referencia al conjunto de creencias personales relativas a la divinidad, vinculadas con lo íntimo y personal de cada uno, al sentido último de la vida, a un Ser superior (Dios) o acciones relacionadas con lo religioso.

Diversas investigaciones realizadas al respecto en pacientes reportan los siguientes efectos de profesar una religión: el control y la recuperación rápida 
de la ansiedad y la depresión (Irwin et al., 1990, citados en Navas et al., 2006), menor probabilidad de suicidio y control de los niveles de tensión arterial (Mueller et al., 2001; Rodrigues Gomes, 2011), afrontamiento positivo de la enfermedad con mejora de la calidad de vida (Hebert et al., 2001), motivación para establecer relaciones personales positivas y mostrar compromiso comunitario, productividad, participación y crecimiento personal (Wink y Dillon, 2003). Permite además afrontar el duelo con un mayor sentido de esperanza (Yoffe, 2007).

Al buscar una explicación de estos efectos, Koening (2012) ha concluido que, cuando se profesan valores religiosos, se tiene confianza en la seguridad de saber que Dios es la fuente de fortaleza, se conocen las respuestas a las preguntas filosóficas fundamentales y estos valores influyen en la regulación metacognitiva de los eventos negativos de la vida y permiten hallar una solución nueva. En adición, la conexión religiosa no se pierde ni se deteriora con la discapacidad física, a diferencia de otros recursos como el trabajo, los pasatiempos o las relaciones.

En relación con los valores religiosos, se han identificado varias necesidades espirituales que deben ser atendidas en las personas (Castelo-Branco et al., 2014; Ramió, 2011; Vilalta, 2010): (a) necesidad de la verdad, (b) encontrar sentido a la existencia y el devenir, (c) necesidad de proyectar su vida más allá del final de la vida, (d) esperanza auténtica, (e) necesidad de estar en paz con Dios, con la naturaleza y con su conciencia, (f) necesidad de prácticas religiosas, de orar, de hablar con su orientador espiritual, revisar sus creencias y respeto hacia ellas, (g) expresar sentimientos y vivencias religiosas, (h) necesidad de mantener la integridad física y espiritual, y (i) amar y ser amado.

Hay pacientes que no manifiestan interés alguno en lo religioso, pero la mayoría tiene una vida espiritual y demanda la atención de este aspecto en su condición de enfermedad y, en especial, cuando sienten temor a la muerte o se enfrentan a ella (Hebert et al., 2001; Mueller et al., 2001). Entonces, las prácticas religiosas del paciente se consideran elementos a los cuales hay que poner atención, conocerlas y respetarlas, por medio del proceso de atención de enfermería, con énfasis en el plan de cuidado que se diseña (Bellido Vallejo y Lendínez-Cobo, 2010). Se recomienda a las enfermeras (a) valorar la condición espiritual del paciente, (b) mostrarse comprensivas y dispuestas a establecer comunicación afectiva, (c) trabajar con el paciente para que ponga en marcha sus propios recursos para satisfacer sus necesidades espirituales, (d) guiar el encuentro en igualdad con el paciente y en respeto máximo, y (e) darle esperanzas verdaderas.

$\mathrm{Al}$ requerírsele brindar cuidado espiritual, el profesional de enfermería debe poseer un equilibrio sano en sus dimensiones para ser capaz de cumplir con ese deber. Como es el responsable del cuidado, su interacción influye directamente sobre el nivel de satisfacción que el paciente alcanza con el servicio y con su disposición a seguir las indicaciones de autocuidado (Rebolledo Oyarzo, 2010). Los profesionales de la enfermería se encargan de situaciones que pueden generar mucho estrés. $\mathrm{Y}$ en los países en desarrollo suelen agregarse más estresores por condiciones laborales inadecuadas, multiempleo y baja calidad de vida (Sierra Leguía y Montalvo Prieto, 2012), 
situaciones que también afectan a las enfermeras dominicanas. En el país se reportan factores estresantes como falta de materiales y equipos para el trabajo, alta proporción de pacientes, relaciones inadecuadas y condiciones ambientales inapropiadas (Peguero de la Rosa, 2016), aunque esto no ocurre en todos los centros de salud de la misma manera.

Pérez-García (2016) explica que, aunque todos estos elementos afectan la calidad del cuidado espiritual, las razones prioritarias por las cuales el personal de enfermería falla en cuidar la espiritualidad del paciente están ligadas a falta de competencia en esta dimensión. La formación profesional exalta el positivismo y niega la dimensión metafísica (Okasha y Okasha, 2012). Pero, atender este asunto, requiere que la enfermera tenga conciencia de su propia espiritualidad, se ejercite en lo espiritual para poder compartir con la persona/familia experiencias reales y rompa el temor a la incapacidad porque puede buscar el apoyo de otro profesional, ya sea el psicólogo o el capellán.

En medio de las presiones, las enfermeras que recurren a los valores religiosos muestran la ecuanimidad y la paciencia necesarias para brindar el cuidado integral a sus pacientes y tienen un alto sentido de responsabilidad compartida (Sierra Leguía y Montalvo Prieto, 2012). Los recursos espirituales que más usan las enfermeras son fundamentalmente religiosos e incluyen rezar/ orar, meditar, entrar en contacto con la naturaleza y relacionarse con un Ser superior (Winterkorn Dezorzi y Oliveira Crosseti, 2008).

Con todas las bondades que los valores religiosos pueden aportar, Altemeyer (1996, citado en Etchezahar y Simkin, 2013; Simkin y Etchevers, 2014) afirma que el autoritarismo puede estar presente en la manifestación de las creencias y producir una actitud cerrada con prejuicio, discriminación y hasta violencia hacia las creencias de los demás. Esto puede prevenirse cuando se toma en cuenta la enfermería transcultural para comprender, interpretar y predecir los fenómenos asistenciales en la profesión de enfermería y guiar su ejercicio hacia una actuación que se amolda a los valores importantes dentro de la cultura en la cual se ofrece el servicio (Leininger y Mcfarland, 2002; Mixer, 2011). Aplicado al caso en estudio, estas declaraciones advierten sobre la necesidad de que el profesional de enfermería evite ser autoritario al trabajar con el cuidado espiritual del paciente; debe respetar su autonomía, permaneciendo abierto a las diferencias socioculturales que este puede mostrar en esta dimensión, sin que esto implique una actuación falsa en función de sus propias creencias. Promover esas competencias es responsabilidad de la institución formadora (Bellido Vallejo y Lendínez Cabo, 2010).

Vargas Peña (2015) encuentra que la interacción armoniosa de seres animados e inanimados en el universo depende de su actitud o negación hacia cinco principios enlazados por el amor: bondad, justicia, libertad, unidad y verdad. Las religiones, en general, independientemente de su cosmovisión, los incluyen en sus enseñanzas con aplicaciones particulares.

Con este marco surge un interés por conocer las reacciones de la enfermera hacia los valores religiosos, que pueden estar afectando su deber de atender la dimensión espiritual de la persona enferma, en el contexto cultural de la República Dominicana, una nación eminentemente religiosa. Entre el $70 \%$ y el 
$90 \%$ de la población dice profesar religiones cristianas como la católica (45\%$66 \%$ ), la evangélica (20\%-17\%) u otras (3\%-12\%), de acuerdo con la Encuesta Demográfica y de Salud (Endesa, 2014) y la Oficina Nacional de Estadísticas y UNICEF (2016).

Suero (2018) reporta que ocho (73\%) de las 11 escuelas universitarias de enfermería activas indican en sus estatutos que su filosofía es cristiana, aunque solo cinco $(45 \%)$ son de instituciones confesionales. La revisión de los planes curriculares y las actividades agendadas en un año académico en estas escuelas indica que (a) la integración de valores y principios éticos-morales es muy débil y (b) los valores cristianos en las instituciones de educación superior con esta filosofía se promueven en actividades extracurriculares o asignaturas filosóficas. Ambas estrategias por lo común se realizan con grupos grandes, donde el acercamiento entre el facilitador y el estudiante es limitado y la mediación se realiza solo con quienes se acercan de manera personal. Pero la integración de esos principios en las asignaturas del plan curricular es superficial o está ausente.

Sin embargo, en las últimas décadas, los fenómenos migratorios han retomado su auge histórico y se recibe demanda de atención de salud de ciudadanos de distintas partes del mundo con diferencias culturales marcadas. Quienes visitan el país de forma esporádica por turismo, negocios o alguna necesidad específica de salud, residen de manera temporal por causa de estudio o trabajo, o se establecen de forma permanente por lazos familiares. Muchos vienen de países donde predomina el cristianismo, como Venezuela y Colombia, pero otros proceden de naciones orientales o islas con religiones del Asia, como China, Corea del Sur, Taiwán y algunos países árabes. En especial, después del terremoto de Haití, es alta la frecuencia de haitianos que buscan atención de salud y muchos de ellos muestran adhesión a religiones espiritistas.

El objetivo de la investigación aquí reportada fue analizar las reacciones de profesionales de enfermería dominicanos activos hacia los valores religiosos, que pueden afectar la calidad del cuidado espiritual que brindan al paciente. La investigación se realizó como parte del diagnóstico previo al desarrollo del modelo de formación profesional de enfermería que se ha denominado deontovalidación.

\section{Método}

El estudio tuvo un abordaje mixto, integrando el método descriptivo transversal, la técnica de aplicación de un test y la observación de casos. En lo cualitativo, se recogieron las reacciones de los sujetos ante los términos de la prueba porque facilitan la comprensión de los resultados obtenidos. La muestra definitiva estuvo compuesta por 203 profesionales de enfermería activos, abordados según su disponibilidad en centros de salud públicos y privados de siete ciudades de la República Dominicana.

El instrumento utilizado fue el Test de Reacción Valorativa (TRV) de García Hoz (1976), modificado por Álvarez Rodríguez (2007), el cual tiene una confiabilidad alta $(\alpha=.94)$. Es una escala tipo Likert de cinco opciones, cuyos valores de respuesta son: a) muy agradable, con valor de dos puntos; b) agradable, con valor de un punto; c) indiferente, con valor 0 ; d) desagradable, con valor de menos un punto; y e) muy desagradable, con valor de menos dos puntos. Este test 
mide la reacción de la persona en diez categorías con 250 reactivos: valores corporales (25), intelectuales (25), afectivos (25), estéticos (25), individuales (25), morales (25), sociales (25), ecológicos (25), instrumentales (25) y religiosos (25). Las categorías de valores corporales, ecológicos e instrumentales fueron suprimidas para hacerla más factible de aplicar en el ambiente laboral y se agregaron siete reactivos para recoger datos demográficos. La categoría de valores religiosos abarca términos del cristianismo, el budismo y el islamismo.

La administración del instrumento se realizó después de verificar que no existía riesgo de perjuicio y de tener el consentimiento firmado de los participantes. Los datos cuantitativos se obtuvieron calculando el índice de correlación $r$ de Pearson y un ANOVA simple. Se aplicó el análisis de contenido a los datos cualitativos, los cuales se triangularon con los hallazgos estadísticos.

\section{Resultados}

Los profesionales que participaron eran, en su mayoría, del sexo femenino $(n=188 ; 93 \%)$ y estaban entre los $21 \mathrm{y}$ 40 años $(n=136,67 \%)$ de edad. Refirieron pertenecer a la religión católica $(n$ $=124,61 \%)$, evangélica $(n=39,19 \%)$, adventista $(n=14,7 \%)$ y pentecostal $(n$ $=6,3 \%)$, entre otras $(n=9,4 \%)$. Tres de ellos $(1 \%)$ no indicaron la religión. Además, 80 sujetos $(39 \%)$ eran egresados de tres escuelas de universidades laicas, 73 (36\%) provenían de cinco escuelas de universidades confesionales y $50(25 \%)$ eran de tres escuelas de enfermería que pertenecen a universidades no confesionales con filosofía cristiana.

La aplicación del test al grupo de 203 egresados de enfermería arrojó puntuaciones en el rango comprendido entre 50 y -50 para cada una de las siete categorías de valores, permitiendo establecer una escala individual y una escala global de valores. En esa escala resultante, los valores religiosos quedaron en la última posición.

El comportamiento de las puntuaciones en la categoría de los valores religiosos se mostró disperso con tendencia hacia las puntuaciones medias. Se obtuvo una frecuencia de $26(13 \%)$ profesionales con puntuación máxima (41-50 puntos), 33 (16\%) en el segundo nivel (31-40 puntos), 76 (37\%) en el tercer nivel (21-30 puntos), 54 (27\%) en el cuarto (11-20 puntos) y 14 (7\%) en el quinto y último (10 puntos o menos).

Esa dispersión se observó relacionada con la religión del sujeto. Para los católicos hubo entre 18 y 20 elementos que les resultaban muy agradables (puntuación entre 36 a 40 puntos) contra unos 5 a 7 que les resultaban indiferentes, desagradables o muy desagradables (entre 0 y -14), lo que les situó en un rango de puntuación total entre 26 y 30 puntos. En el caso de los que pertenecen a religiones protestantes, solo mostraron reacciones muy agradables ante unos 12 a 14 términos (entre 24-28 puntos para sus puntuaciones máximas) y de 11 a 13 (entre 0 y -26) que oscilaron de indiferentes a muy desagradables.

En el análisis de ANOVA, no se observaron diferencias significativas de las puntuaciones por categoría de valores entre los grupos formados por las categorías de las variables edad, institución de egreso o religión. Pero se hallaron diferencias significativas en las puntuaciones de 16 de los 25 términos de la categoría de valores religiosos y en las puntuaciones del $48 \%$ de los términos incluidos en la escala completa del test de apreciación valorativa, entre las distintas religiones cristianas en el contexto dominicano. 


\section{Sentimientos y reacciones a la prueba}

Mientras se aplicaba la prueba, llamó la atención el conjunto de reacciones y la manifestación de sentimientos que externaron las participantes y que no se observaron en los 15 (7\%) hombres que llenaron dicho test. Las enfermeras solían estar calladas y concentradas mientras respondían los reactivos y de vez en cuando dejaban escapar una que otra expresión o hacían una pregunta, algunas más que otras. Pero al llegar a la sección de valores religiosos era común que manifestaran buen humor con jovialidad y risas o gestos despectivos frente a términos que les eran desconocidos o les resultaban poco agradables. Este aspecto no estaba contemplado en la investigación. Sin embargo, por su repetición en sujetos de distintos centros de salud de localidades diferentes, y su relación con los resultados en la categoría de valores religiosos, se consideró importante reportarlo, considerándolo como una especie de serendipia que era útil para comprender los resultados del test.

Algunas enfermeras usaron expresiones despectivas coloquiales para referirse a términos religiosos que no les eran familiares y otras mostraban desconcierto. Ambas reacciones revelaban que existía desconocimiento de esos elementos. Entre los términos por cuyo significado las participantes preguntaron más y tuvieron más reacciones de rechazo estuvieron: Alá, mezquita y Mahoma. Entre aquellos que causaron reacciones entre los no católicos están: confesarse, catequesis y misa. Los términos que provocaron reacciones positivas fueron: Dios, Biblia, Jesucristo, oración y misionero.

Los términos del budismo no suscitaron comentarios audibles, reacciones ni preguntas sobre su significado, aunque fueron marcados en reacción desagrada- ble. Esto lleva a percibir que su significado era conocido por las egresadas.

Se notó que la prueba toma en consideración la religión cristiana con mayor cantidad de términos del enfoque católico, hallándose siete en total que fueron indicados como de reacción muy desagradable por la mayoría de los egresados no católicos (catequesis, clase de religión, cuaresma, misa, obispo, papa y sacerdote), además de los cinco términos de religiones orientales (Alá, budismo, Corán, Mahoma, mezquita) que también recibieron poca o ninguna aceptación de la mayoría global, algunos con diferencias significativas por religión y otros no.

\section{Discusión}

Los egresados de las escuelas de enfermería que participaron en la prueba mostraron, en la característica religión, una distribución similar a la que presentan las estadísticas en los censos nacionales (Endesa, 2014), pues, el 97\% indicó pertenecer a una religión cristiana con predominio del catolicismo (61\%). El $60 \%(n=123)$ del grupo es egresado de las escuelas que pertenecen a universidades con filosofía cristiana, de los cuales, 82 (40\%) procede de universidades confesionales. En estas instituciones, la religión se promueve, por lo general, separada de las clases regulares en asignaturas específicas limitadas a un momento de la carrera o en actividades generales (Suero, 2018). Esto deja la integración de los valores religiosos a merced del modelaje que haga el maestro, el cual puede tener objetivos distintos a los de la escuela.

La categoría de valores religiosos resultó en la más baja posición de la escala de valores de los profesionales observados, con tan solo un $13 \%$ de los sujetos 
en la puntuación máxima. De primera impresión, las bajas puntuaciones en esta categoría de valores pueden interpretarse como una presencia débil de valores religiosos en los enfermeros observados. Sin embargo, el análisis de las reacciones de los egresados en el Test de Reacción Valorativa para los términos de dicha categoría, revela lo contrario.

De los 25 reactivos de términos religiosos, cinco son propios de religiones orientales; estos fueron señalados por el grupo como de reacción entre muy desagradable e indiferente, y tres de ellos marcaron diferencias significativas. Otros ocho términos religiosos resultaron ser agradables a los católicos y desagradables a los no católicos, siempre con oscilaciones que resultaron significativas para todos ellos. Y cinco términos fueron agradables a los no católicos y lo opuesto para los católicos.

Estos resultados explican la dispersión de frecuencias en las puntuaciones de la categoría de valores. Para los católicos, según las diferencias de opinión que mostraron, hubo entre 18 y 20 términos que les resultaban muy agradables (puntuación entre 36 a 40 puntos), contra un rango de 5 a 10 que les resultaban indiferentes, desagradables o muy desagradables (entre 0 y -20 puntos), lo cual arrojó un rango de puntuación total promedio entre 26 y 30 puntos. En el caso de los que pertenecen a religiones protestantes, solo hay en la prueba entre 12 y 14 términos que indicaron como de reacción muy agradable (unos 24-28 puntos) y de 11 a 13 (entre 0 y -26) que oscilaron de indiferentes a muy desagradables. Por lo tanto, las puntuaciones obtenidas por los profesionales de enfermería observados en esta categoría implican una presencia fuerte de valores religiosos pero referidos a sus creencias.
Suero y Ferriol (2019) han reportado que, en la escala de valores de los egresados, las posiciones de las categorías quedaron en este orden: valores individuales $(n=145,71 \%)$, valores afectivos $(n=114,56 \%)$, valores intelectuales $(n$ $=77,38 \%)$, valores morales $(n=75$, $37 \%)$, valores sociales $(n=48,24 \%)$, valores estéticos $(n=38,19 \%)$ y valores religiosos $(n=26,13 \%)$.

$\mathrm{Al}$ observar las reacciones de los egresados a los términos de la categoría religiosa, unidos a las posiciones de las categorías en la escala global, se infiere que la explicación a la ocupación de la última posición en la escala de valores para la categoría de religiosos es un fuerte arraigo a las creencias religiosas que se han aceptado y el rechazo de las que no pertenecen a ese conjunto dogmático. Esto así, porque los profesionales se mostraron definidos en sus creencias, sin importar la religión que profesan, la institución de donde egresan o su edad, pero de igual forma tienen elevados los valores individuales.

Este es un dato positivo desde el punto de vista del principio de libertad y del principio de unidad (Vargas Peña, 2015), pues testifica que estos profesionales ejercen su individualidad al escoger y unirse a la comunidad religiosa que desean. Además, habla de que estos profesionales poseen un conocimiento experiencial de la dimensión espiritual que se identifica como necesario para poder dar cuidado espiritual (Sierra Leguía y Montalvo Prieto, 2012).

Sin embargo, puede presentar dificultades hacia los principios de bondad y justicia, si el desagrado que manifiestan hacia las creencias del otro llegara a romper los límites del respeto y la dignidad ajena, aun cuando sus puntuaciones en valores afectivos fueron muy buenas, 
ocupando esta categoría el segundo nivel de la escala. Como han advertido Etchezahar y Simkin (2013) y Simkin y Etchevers (2014), el autoritarismo puede llevar a reacciones que niegan estos principios en contraposición al deber humanitario de la enfermería.

Las expresiones y sentimientos que manifestaron los sujetos frente a los valores religiosos presentados por la prueba indicaron que existe cierto grado de limitación, desconocimiento y rechazo cultural que contribuye, también, con las puntuaciones bajas que obtuvieron los sujetos y con la posición de la escala en la cual se ubicó esta categoría. El desconocimiento de elementos de religiones árabes contrastado contra el dominio de las religiones asiáticas destaca la importancia de la práctica contra la teoría para la fijación del conocimiento. Ambas culturas están incluidas en el currículo dominicano para las ciencias sociales en el nivel secundario o preuniversitario, pero los dominicanos están mucho más relacionados con la cultura china y la japonesa cuyos nativos están más ligados en su medio. Y las expresiones despectivas y peyorativas al comprender el significado de los términos que ignoraban, podrían estar ligadas a una concepción de esas culturas conectada con hechos negativos en manifestaciones terroristas, reportadas en noticieros nacionales e internacionales, puesto que se observaron hacia términos del Islam pero no hacia la mención del budismo.

La manera de promover los valores religiosos desde las escuelas de enfermería y sus instituciones, tampoco ayuda a mejorar el cuadro. Las actitudes observadas se corresponden con una promoción de valores religiosos generalizada y dogmática, observada en las instituciones formadoras (Suero, 2018), que no se detiene lo suficiente en el acercamiento al estudiante para trabajar sus necesidades de tolerancia, compañerismo y respeto de las creencias del otro. Esto se puede atribuir a dedicación de tiempo limitada por parte de los administradores de las escuelas, los docentes $\mathrm{y}$ los propios estudiantes, $\mathrm{y}$, una integración de valores y principios poco intencional y no sistemática (Suero, 2018). También podría estar relacionado con el tipo de modelaje que estén ofreciendo los docentes y el personal administrativo de las escuelas, en los distintos ambientes educativos (Suero y Ferriol, 2020).

Leininger y Mcfarland (2002), al igual que Mixer (2011), llaman la atención a la necesidad de tomar en cuenta la cultura como estrategia para brindar un cuidado de calidad y poder predecir los fenómenos de la atención. Esto debe trabajarse en la formación profesional para que la enfermera cuente con estrategias para mantener su autenticidad religiosa, mientras cuida la espiritualidad del paciente con trato amable y respeto hacia las creencias de la persona/familia/comunidad.

\section{Conclusiones}

Las reacciones frente a los valores religiosos en el personal de enfermería dominicano están condicionadas por una presencia fuerte de apego a las creencias que han aceptado y rechazo e indiferencia hacia las creencias del otro. Esto, unido a una priorización de los valores individuales puede ser obstáculo para brindar cuidado espiritual dentro de su contexto a personas con creencias religiosas de otras culturas, aunque el nivel de valores afectivos que muestran estos profesionales es muy bueno.

Los valores espirituales son un factor importante en la atención de salud, tanto 


\section{SUERO DE LORA Y FERRIOL SÁNCHEZ}

para el cuidado espiritual del paciente como para el autocuidado del personal de enfermería. Al igual que los demás valores, no pueden ser enseñados como simples teorías enajenadas de la práctica real. Es en el hacer donde el acompañamiento, ya sea en la formación o en el seguimiento laboral, puede notar actitudes que revelan interpretaciones desviadas y orientar hacia el desarrollo de competencias actitudinales y procedimentales correctas que capaciten para afrontar las demandas de cuidado espiritual de personas con culturas distintas a las propias.

Las escuelas de enfermería no deben dejar este aprendizaje al azar, a merced de lo que el docente por sí mismo decida promover en forma consciente o inconsciente. Deben analizar la forma de hacer una integración intencional y sistemática para orientar las actitudes hacia los valores de la bondad en el cuidado espiritual en un marco transcultural.

\section{Referencias}

Álvarez Rodríguez, J. (2007). Test axiológico: Un instrumento para detectar valores. Revista Portuguesa de Pedagogía, 41(1), 157-177. https://doi.org/10.14195/1647-8614_41-1_9

Bellido Vallejo, J. C. y Lendínez Cobo, J. F. (Eds.). (2010). Proceso enfermero desde el modelo de cuidados de Virginia Henderson y los Lenguajes NNN. Colegio Oficial de Enfermería de Jaén.

Castelo-Branco, M. Z., Brito, D. y Fernandes-Sousa, C. (2014). Necessidades espirituais da pessoa doente hospitalizada: revisão integrativa. Aquichán, 14(1), 100-108. https://doi.org/10.5294/aqui.2014.14.1.8

Consejo Internacional de Enfermería. (2012). Código deontológico internacional del CIE para la profesión de enfermería. http://www.ee.lafe.san.gva.es/pdfs/icncodesp.pdf

Encuesta demográfica y de salud, República Dominicana. (2014). Ministerio de Salud Pública. https:// dhsprogram.com/pubs/pdf/PR43/PR43.pdf

Etchezahar, E. y Simkin, H. (2013). Religiosidad, espiritualidad y escepticismo: La mediación del autoritarismo. Subjetividad y Procesos Cognitivos, 17(2), 48-58. http://dspace.uces.edu.ar:8180/xmlui/ handle/ 123456789/2397

García Hoz, V. (1976). "Test” de reacción valorativa. Bordón, 214, 245-270. https://dialnet.unirioja.es/ servlet/articulo? codigo $=2346305$

Henderson, V. (1961). Principios básicos de los cuidados de enfermería. CIE-OPS.

Hebert, R. S., Jenckes, M. W., Ford, D. E., O'Connor, D. R. y Cooper, L. A. (2001). Patient perspectives on spirituality and the patient-physician relationship. Journal of General Internal Medicine, 16(10), 685-692. https://doi.org/10.1111/j.1525-1497.2001.01034.x

Koening, H. G. (2012). Religion, spirituality and health: The research and clinical implications. International Scholarly Research Notices, Article ID 278730. https://doi.org/10.5402/2012/278730

Leininger, M. y McFarland, M. R. (2002). Transcultural Nursing: Concepts, theories, research and practice (3a ed.). McGraw-Hill.

Mixer, S. (2011). Use of the culture care theory to discover nursing faculty care expressions, patterns, and practices related to teaching culture care. Online Journal of Cultural Competence in Nursing and Healthcare, 1(1), 3-14. http://www.ojccnh.org/v1n1a1.shtml

Mueller, P. S., Plevak, D. J. y Rummans, T. A. (2001). Religious involvement, spirituality and medicine: Implications for clinical practice. Mayo Clinic Proceedings, 76, 1225-1235. https://doi. org/10.4065/76.12.1225

Navas, C., Villegas, H., Hurtado, R. y Zapata, D. (2006). La conexión mente-cuerpo-espíritu y su efecto en la promoción de la salud en pacientes oncológicos. Revista Venezolana de Oncología, 18(1), 28-37. http:// ve.scielo.org/scielo.php?script=sci_arttext\&pid=S079805822006000100006\&lng=es\&nrm=iso\&tlng=es

Oficina Nacional de Estadística y UUNICEF. (2016). Encuesta Nacional de Hogares de Propósitos Múltiples-ENDESA. Encuesta de indicadores múltiples por conglomerados 2014, Informe Final. Oficina Nacional de Estadística, República Dominicana.

Okasha, A. y Okasha, T. (2012). Religión, espiritualidad y el concepto de enfermedad mental. Actas Españolas de Psiquiatría, 40(2), 73-79. https://www.actaspsiquiatria.es/repositorio/suplements/14/ ESP/14-ESP-378649.pdf 


\section{VALORES RELIGIOSOS EN PROFESIONALES DE ENFERMERÍA}

Orem, D. E. (1959). Guides for developing curricula for the education of practical nurses. Office of Education.

Peguero de la Rosa, Y. (2016). Influencia del estrés en el personal de enfermería de atención directa en la unidad de emergencias del Hospital Regional Dr. Antonio Musa 2014-2015. [Tesis de grado inédita]. Universidad Central del Este, República Dominicana.

Pérez-García, E. (2016). Enfermería y necesidades espirituales en el paciente con enfermedad en etapa terminal. Enfermería: Cuidados Humanizados, 5(2), 41-45. https://doi.org/10.22235/ech.v5i2.1286

Puentes Silva, Y. M., Urrego Barbosa, S. C. y Sánchez Pedraza, R. (2015). Espiritualidad, religiosidad y enfermedad: Una mirada desde mujeres con cáncer de mama. Avances en Psicología Latinoamericana, 33(3), 481-495. https://doi.org/10.12804/apl33.03.2015.08

Ramió, A. (2011, 12-13 de mayo). Necesidades espirituales y atención profesional (Sesión de Conferencia). IX Jornada Nacional de la SECPAL. Espiritualidad en clínica, Palma de Mallorca, España.

Rebolledo Oyarzo, D. P. (2010). Autocuidado en los estudiantes de la carrera de enfermería de la Universidad del Bío-Bío: un análisis según el modelo de autocuidado de Dorotea Orem [Tesis de licenciatura, Universidad Autral de Chile]. http://cybertesis.uach.cl/tesis/uach/2010/fmr292a/doc/ fmr292a.pdf

Rodrigues Gomes, A. M. (2011). La espiritualidad ante la proximidad de la muerte. Enfermería Global, 10(2). https://doi.org/10.4321/S1695-61412011000200019

Salgado, A. C. (2014). Revisión de estudios empíricos sobre el impacto de la religión, religiosidad y espiritualidad como factores protectores. Propósitos y Representaciones, 2(1), 121-129. https://doi. org/10.20511/pyr2014.v2n1.55

Sierra Leguía, L. y Montalvo Prieto, A. (2012). Bienestar espiritual de enfermeras y enfermeros en unidades de cuidado intensivo. Avances en Enfermería, 30(1), 64-74. https://revistas.unal.edu.co/index.php/ avenferm/article/view/35438/36509

Simkin, H. y Etchevers, M. (2014). Religiosidad, espiritualidad y salud mental en el marco del Modelo de los Cinco Factores de la Personalidad. Acta Psiquiátrica y Psicológica de América Latina, 60(4), 264275. https://www.aacademica.org/hugo.simkin/22.pdf

Suero, A. R. (2018). Modelo curricular para la integración de principios éticos en la formación de enfermería en las escuelas universitarias dominicanas [Tesis doctoral no publicada]. Universidad Internacional Iberoamericana, República Dominicana.

Suero Moreta, A. R., y Ferriol, F. (2020). Deontovalidación en la relación docente-estudiante. Revista Electrónica de Investigación y Docencia, 23, 9-23. https://doi.org/10.17561/10.17561/reid.n23.1

Suero Moreta, A. R. y Ferriol, F. (2019). Escala de valores en profesionales de enfermería activos de República Dominicana. MLS Educational Research, 3(2), 87-100. https://doi.org/10.29314/mlser. v3i2.186

Vargas Peña, D. (2015). El sistema ético-moral: principios, valores e indicadores. Ápeiron.

Vilalta, A. (2010). Evaluación de las necesidades espirituales de pacientes diagnosticados de cáncer avanzado y terminal. [Tesis doctoral, Universitat de Lleida]. Tesis Doctorals in Xarxa. https://www. tdx.cat/handle/10803/8072\#page $=1$

Watson, J. (2015). Jean Watson's theory of human caring. En M. C. Smith y M. E. Parker (Eds.), Nursing theories and nursing practice (4⿳亠丷⿵冂⿰丨丨 ed., pp. 321-339). F. A. Davis.

Wink, P. y Dillon, M. (2003). Religiousness, spirituality and psychosocial functioning in late adulthood: Findings from a longitudinally study. Psychology and Aging, 18(4), 916-924. https://doi. org/10.1037/0882-7974.18.4.916

Winterkorn Dezorzi, L. y Oliveira, M. G. (2008). La espiritualidad en el cuidado de sí para profesionales de enfermería en terapia intensiva. Revista Latinoamericana de Enfermagem, 16(2), 212-217. https://doi. org/10.1590/S0104-11692008000200007

Yoffe, L. (2007). Efectos positivos de la religión y espiritualidad en el afrontamiento de duelos. Psicodebate, 7, 193-206. https://doi.org/10.18682/pd.v7i0.435

Recibido: 20 de abril de 2020

Revisado: 15 de junio de 2020

Aceptado: 6 de julio de 2020 